\title{
FATORES DE RISCO PARA O DESENVOLVIMENTO DE LESÃO POR PRESSÃO EM PACIENTES
} CRÍIICOS

Carolina Ottoํ․, Beatriz Schumacher², Luiz Paulo De Lemos Wiese ${ }^{3}$, Carlos Ferro², Raquel Antonacci Rodrigues²

Objetivo: Identificar a relação entre os fatores de risco para o desenvolvimento de lesão por pressão e determinar sua incidência em pacientes críticos. Metodologia: Estudo descritivo de abordagem quantitativa, que avaliou pacientes internados em uma unidade de terapia intensiva, durante o periodo de 1으 de maio a 30 de outubro de 2015 . Resultados: Participaram do estudo 59 pacientes, destes 29 desenvolveram lesão por pressão, incidência de 49,2\%. Quanto às variáveis demográficas e clínicas, houve predominância do gênero masculino, média de idade $(46,9 \pm 19,8)$, associação estatística ( $p=<0,001)$ para maior tempo de internação, ventilação mecânica, sedação, balanço hídrico positivo e uso de antibióticos, Escala de Braden, no escore risco elevado e risco leve e Simplified Acute Physiology Score $(p=0,09)$. Conclusão: As lesões apresentam multicausalidade, sugerindo-se que a prevenção e tratamento sejam realizados através da educação nos serviços com fortalecimento dos protocolos.

Descritores: Lesão por Pressão; Enfermagem; Unidade de Terapia Intensiva; Fatores de Risco; Protocolos; Cuidados Intensivos.

\section{RISK FACTORS FOR THE DEVELOPMENT OF PRESSURE LESION IN CRITICAL PATIENTS}

Objective: To identify a relationship between the risk factors for pressure lesion development and to determine its incidence in critical patients. Methodology: This was a descriptive study of a quantitative approach, which evaluated patients hospitalized in an intensive care unit during the period from May lst to October 30th , 2015. Results: 59 patients took part in the study, 29 of them developed pressure lesion, a 49,2\% of incidence. As for demographic and clinical variables, there was a predominance of male gender, mean age (46.9 \pm 19.8), statistical association $(p=<0.001)$ for longer hospitalization, mechanical ventilation, sedation, positive water balance and use of antibiotics, Braden Scale, did not score high risk and light risk and simplified acute physiology score $(p=0.09)$. Conclusion: The lesion show multi-causality, it is suggested that prevention and treatment are carried out through education in services with strengthening protocols.

Descriptors: Pressure Lesion; Nursing; Intensive Care Units; Risk Factors; Protocols; Intensive Care.

\section{FACTORES DE RIESGO PARA EL DESARROLLO DE LESIONES POR PRESIÓN EN PACIENTES CRÍTICOS}

Objetivo: Identificar la relación entre los factores de riesgo para el desarrollo de lesiones por presión y determinar su incidencia en pacientes críticos. Metodología: Estudio descriptivo de abordaje cuantitativo, que evaluó a pacientes internados en una unidad de terapia intensiva, durante el período del 1 de mayo al 30 de octubre de 2015. Resultados: Participaron del estudio 59 pacientes de estos 29 desarrollaron lesiones por presión, 49,2\%. En cuanto a las variables demográficas y clínicas, hubo predominio del género masculino, promedio de edad $(46,9 \pm 19,8)$, asociación estadística $(p=<0,001)$ para mayor tiempo de internación, ventilación mecánica, sedación, balance hídrico positivo y uso De antibióticos, Escala de Braden, en el score de riesgo elevado y riesgo leve y Simplified Acute Physiology Score ( $p=0,09)$. Conclusión: Las lesiones presentan multicausalidad, se sugiere que la prevención y tratamiento sean realizados a través de la educación en los servicios con fortalecimiento de los protocolos.

Descriptores: Lesiones por presión; Enfermería; Unidad de terapia intensiva; Factores de riesgo; Protocolos; Cuidados Intensivos.

1Hospital Municipal São José de Joinville,SC,Brasil.

${ }^{2}$ Faculdade Bom Jesus/IELUSC - Joinville, SC

${ }^{3}$ Universidade da Região de Joinville - UNIVILLE - Joinville, SC.

Autor correspondente: Carolina Otto: . Email: carol.otto@yahoo.com.br 


\section{INTRODUÇÃO}

As Unidades de Terapia Intensiva (UTI) apresentam pacientes com características peculiares em decorrência de sua gravidade clínica, instabilidade hemodinâmica dos sistemas orgânicos ${ }^{(1,2)}$.

Essas condições requerem mecanismos de suporte à vida como o uso de ventilação mecânica, sedação contínua, drogas vasoativas, monitorizações e diversos tipos de dispositivos como cateteres, drenos e sondas. Por isso, os pacientes estão mais expostos e vulneráveis a alterações no processo de manutenção da integridade da pele, favorecendo ao desenvolvimento de Lesão por Pressão (LPP) ${ }^{1-3}$.

Durante a internação na UTI, existem situações que comprometem a perfusão tissular da pele, como: redução da pressão sanguínea decorrente dealterações cardiovasculares, sindrome da resposta inflamatória sistêmica (SIRS), choque séptico, choque hemorrágico, uso de fármacos e instabilidade hemodinâmica ${ }^{1,2}$.

Em qualquer uma dessas situações, o paciente crítico sofre com as alterações do fluxo sanguíneo para a área que está sobre pressão, comprometendo a oxigenação e a nutrição dos tecidos naquele naquela região, podendo levar ao desenvolvimento de isquemia, hipóxia, edema e necrose tecidua ${ }^{(2,3}$.

Vários fatores de risco estão associados com o desenvolvimento de LPP em pacientes críticos, tais como: alterações do nível de consciência, déficit nutricional, pressão extrínseca associada à idade avançada, umidade, imobilidade no leito, período prolongado de internação, perfusão tecidual diminuída, uso de drogas vasoativas, sepse, sedação e as comorbidades como diabetes mellitus e doença vascular ${ }^{1,2,5}$.

As LPP são classificadas de acordo com seis categorias, conforme quadro $\mathrm{Ol}$.

Quadro 01: Classificação da Lesão por Pressão

\begin{tabular}{|c|c|}
\hline Categoria & Descrição \\
\hline Categoria I & $\begin{array}{l}\text { Lesão eritematosa não branqueável, em pele intacta nas áreas de } \\
\text { proeminência óssea. }\end{array}$ \\
\hline Categoria II & $\begin{array}{l}\text { Perda parcial da espessura da derme, apresentando-se de forma } \\
\text { abrasiva, bolhosa, sem tecido desvitalizado. }\end{array}$ \\
\hline Categoria III & $\begin{array}{l}\text { Perda cutânea total, acometendo área de tecido subcutâneo, as } \\
\text { lesões podem ser cavitadas com fistula e apresentar tecido } \\
\text { desvitalizado. }\end{array}$ \\
\hline Categoria IV & $\begin{array}{l}\text { Perda total da espessura do tecido com exposição óssea, de } \\
\text { músculos, tendões ou cápsulas das articulações. }\end{array}$ \\
\hline $\begin{array}{l}\text { Categoria não } \\
\text { graduável/ } \\
\text { inclassificável }\end{array}$ & $\begin{array}{l}\text { Perda total dos tecidos, com a profundidade preenchida por } \\
\text { tecido necrosado. }\end{array}$ \\
\hline $\begin{array}{l}\text { Categoria } \\
\text { suspeita de } \\
\text { lesão tissular } \\
\text { profunda }\end{array}$ & $\begin{array}{l}\text { Lesões com áreas vermelho-escuras, flictena com sangue, } \\
\text { provocadas por danos no tecido mole subjacente resultantes de } \\
\text { pressão e ou cisalhamento. }\end{array}$ \\
\hline
\end{tabular}

8 | Enferm. Foco 2019; 10 (1): 07-11
Fonte: National Pressure Lesion Advisory Panel, European Pressure Lesion Advisory Panel, Pan Pacific Pressure Injury Alliance ${ }^{4}$.

O desenvolvimento das LPP é um problema mundial em todos os níveis assistenciais de saúde, afetando pessoas de todos os grupos etários e resultando em encargos financeiros significativos para os sistemas de saúde, com aumento no investimento em materiais, equipamentos, fármacos, intervenções cirúrgicas e do tempo de internação(1.5).

O presente estudo teve por objetivo identificar a relação entre os fatores de risco para o desenvolvimento de LPP e determinar sua incidência em pacientes graves internados em uma UTI.

\section{METODOLOGIA}

\section{Tipo de estudo}

Estudo descritivo, de abordagem quantitativa.

\section{Participantes da pesquisa}

Foram incluídos no estudo todos os pacientes com idade superior ou igual a 18 anos que não apresentaram LPP no momento da admissão, no período de 1 으 de maio a 30 de outubro de 2015. Foram excluidos os pacientes que possuíam LPP no momento da admissão e tempo de internação menor que 48 horas.

\section{Local do estudo}

Desenvolvido em uma UTI Geral, composta por 8 leitos, de um hospital público do norte do estado de Santa Catarina (SC).

\section{Coleta dos dados}

A primeira etapa da coleta de dados consistiu na avaliação das variáveis demográficas e clínicas no momento da admissão na UTI: gênero, cor de pele, idade, comorbidades, diagnósticos clínicos, Simplified Acute Physiology Score (SAPS III), instrumento composto de 20 variáveis mensuráveis que avalia o prognóstico de gravidade dos pacientes na UTI e Escala de Risco de Braden (em até 48 horas da admissão), ferramenta de avaliação sistemática dos riscos para desenvolvimento de LPP em pacientes críticos. Esses dados foram coletados com informações obtidas do prontuário eletrônico.

A segunda etapa foi composta pelas seguintes variáveis clínicas: permanência na UTI (dias); sedação contínua (dias); ventilação mecânica (dias); balanço hídrico positivo (dias); uso de antibiótico (dias); uso de corticoides (dias); Escore da Escala de Glasgow; dose máxima e média de noradrenalina (mcg/ kg/min); presença de LPP; localização da LPP; classificação 
da LPP (conforme categoria I, II, III, IV, não graduável/ inclassificável e suspeita de lesão tissular profunda).

Esses dados foram coletados diariamente, através de protocolos institucionais de prevenção, análise do prontuário eletrônico e das prescrições médicas e de enfermagem.

\section{Procedimentos de análise dos dados}

Os dados foram anali-sados seguindo as etapas de tabulação utilizando programa Software Microsoft Office Excel 2010. Em seguida, foi realizada a sintese das principais estatísticas: cálculo de incidência e prevalência das LPP. As variáveis com distribuição normal foram apresentaᄀdas com média e desvio padrão; as variáveis contínuas foram comparadas com o teste t-Student e as variáveis categóricas foram comparadas com Qui-quadrado de Pearson. Valores de $p<0,05$ foram considerados significativos. Quando as comparações das variáveis categóricas resultaram significativas, exibiu-se o Odds Ratio (OR) com o respectivo Intervalo de Confiança (IC95\%).

Procedimentos éticos

O estudo foi aprovado pelo Comitê de Étiনca sob número de aprovação CAAE 40923014.8.0000.5362.

\section{RESULTADOS}

Foram admitidos 88 pacientes na unidade no período da realização da pesquisa; desses, 29 foram excluidos por apresentar LPP no momento da admissão ou permanecer por menos de 48 horas. Dos 59 pacientes incluídos, 29 desenvolveram e 30 não desenvolveram LPP, correspondendo a uma incidência de 49,2\%.

A avaliação desses 59 pacientes incluídos no estudo foi realizada no período de 58 dias, sendo que a média de internação na UTI dos pacientes que desenvolveram LPP foi de 24,6 dias e o tempo médio para o surgimento de LPP foi de 2,7 dias. Foram identificadas 55 LPP; as áreas mais frequentes foram a região sacral 17 (30,9\%), occipital 12 (21,8\%) e calcâneos 11 (20\%), e as categorias mais prevalentes o nível I e II.

Na tabela 1, é apresentada a distribuição das variáveis demográficas e clínicas investigadas segundo presença ou ausência de LPP.
Tabela 1: Distribuição das variáveis demográficas e clínicas

\begin{tabular}{|c|c|c|c|}
\hline Variáveis & $\begin{array}{l}\text { Presença de } \\
\text { LPP (N=29) } \\
\text { Média ( } \pm \text { DP) }\end{array}$ & $\begin{array}{l}\text { Ausếncia de } \\
\text { LPP (N=30) } \\
\text { Média ( } \pm \mathrm{DP})\end{array}$ & Valor $\mathbf{p}$ \\
\hline Sexo masculino, n (\%) & $22(75,8)$ & $13(43,3)$ & 0,01 \\
\hline Cor de pele: Brancos, $n(\%)$ & $21(72,4)$ & $24(80)$ & - \\
\hline Pretos, n (\%) & $6(20,6)$ & $4(13,3)$ & - \\
\hline Pardos, n (\%) & $2(6,8)$ & $2(6,6$ & - \\
\hline Idade, anos & $46.9 \pm 19.8$ & $50,3 \pm 14,2$ & $<0.001$ \\
\hline SAPS III admissão & $55,3 \pm 22,4$ & $39 \pm 23$ & 0.09 \\
\hline Permanência na UTI, dias & $24,6 \pm 15,1$ & $10.1 \pm 4.8$ & $<0.001$ \\
\hline Sedação contínua, dias & $13,7 \pm 8,6$ & $3,6 \pm 3,9$ & $<0,001$ \\
\hline Ventilação Mecânica, diass & $21,9 \pm 13,7$ & $5,6 \pm 4,9$ & $<0,001$ \\
\hline Balanço húdrico positivo, dias & $16,4 \pm 9,4$ & $6,3 \pm 4,1$ & $<0,001$ \\
\hline Antibiótico, dias & $15.7 \pm 10.2$ & $6.3 \pm 4.8$ & $<0.001$ \\
\hline Escala de Glasgow & $7.7 \pm 4.4$ & $11,3 \pm 4,5$ & 0.003 \\
\hline Corticoide, dias & $4,3 \pm 6.8$ & $2,1 \pm 4,0$ & 0.135 \\
\hline Noradrenalina: Dose máxima* & $0,4 \pm 0,3$ & $0,3 \pm 0,4$ & 0,248 \\
\hline Noradrenalina: Média & $0,2 \pm 0,1$ & $0,1 \pm 0,2$ & 0,75 \\
\hline \multicolumn{4}{|l|}{ Escala de Braden admissão } \\
\hline Risco elevado, n (\%) & $22(75.9)$ & $7(23.3)$ & $<0.001$ \\
\hline Risco alto, $n(\%)$ & $7(24.1)$ & $11(36.6)$ & 0.29 \\
\hline Risco moderado, n (\%) & $0(0)$ & $3(10)$ & 0.08 \\
\hline Risco leve, n (\%) & $0(0)$ & $9(30)$ & $<0,001$ \\
\hline
\end{tabular}

LPP - Lesão por Pressão; SAPS III - Simplified Acute Physiology Score; ${ }^{*}$ Dose de noradrenalina apresentada em $\mathrm{mcg} / \mathrm{kg} / \mathrm{min}$; Resultados expressos em número (\%) ou média \pm desvio padrão.

Quanto às variáveis demográficas e clínicas dos pacientes com LPP versus pacientes sem LPP, é possivel observar que o gênero masculino foi predominante. Nos dois grupos, verificou-se predomínio da cor de pele branca e a média de idade dos pacientes com LPP foi menor. A média do SAPS III na admissão dos pacientes com LPP foi superior aos sem LPP.

Quanto maior o tempo de internação, maior o risco de LPP. Desta forma, é possivel considerar que os pacientes submetidos a um período prologado de uso de ventilação mecânica, sedação contínua, dias de balanço hídrico positivo e uso de antibióticos estão mais suscetiveis a desenvolver LPP.

Para o escore da Escala de Risco de Braden, os resultados mostraram a existência estatística significativa na predição da avaliação de risco de LPP, no escore risco elevado e risco leve. 
Em relaçãoàvariável presença de LPP, segundo diagnóstico de internação na UTI, observa-se o predomínio de doenças traumáticas: 14 (48,2\%) nos pacientes que desenvolveram LPP e $8(26,6 \%)$ nos sem LPP, ( $p=0,08)$ e Odds Rati (OR): 2,6 e Intervalo de Confiança de 95\% - (IC95\%): 0,86 - 7,6. Os outros diagnósticos de internação na UTI não demonstraram associação estatística.

\section{DISCUSSÃO}

Os resultados da pesquisa demonstram alta incidência de LPP 49,2\%. No Brasil, estudos demonstram incidência de LPP em UTI com referência em traumatologia de 37\% e 59,5 \% (5).

Quanto à localização das LPP evidenciadas, as áreas mais frequentes foram a região sacral, occipital e calcâneos. Em outros dois estudos, desenvolvidos com pacientes críticos, os autores constataram que os principais locais de desenvolvimento de LPP foram a região sacral e calcâneos, consideradas locais de apoio quando o paciente está em decúbito dorsal ou lateral (comuns entre pacientes críticos) $(1,6)$. Em relação às categorias, o nivel I e II são as mais prevalentes, confirmando os resultados obtidos entre os pacientes do presente estudo e dados da literatura atual.

A maioria dos pacientes com LPP foram do sexo masculino. Em um estudo realizado em três UTIs de São Paulo, referência em pacientes politraumatizados, identificou-se (56,3\%) pacientes do sexo masculino.6 Em uma pesquisa realizada em uma UTI que atende pacientes cirúrgicos e clínicos, houve equilibrio entre os sexos, com (51\%) mulheres e (49\%) homens 5 .

A população masculina predominante encontrada no estudo é condizente com as características do local da pesquisa, um hospital público de grande porte especializado em trauma, que atende pessoas em sua maioria vítimas de acidentes de trânsito e indivíduos do gênero masculino.

As doenças traumáticas (48,2\%) foram o principal diagnóstico clínico de internação na UTI dos pacientes com LPP. Os indivíduos vítimas de trauma estão relacionados a fatores etiológicos, tais como: acidentes de trânsito e de trabalho, tentativas de suicídio e violência interpessoal, quando apresentam risco de morte iminente e requerem assistência em unidades especializadas como UTI . No Brasil, no ano de 2016, as causas externas corresponderam à segunda causa de morbidade hospitalar em homens ${ }^{8}$.

Em relação à idade do grupo que desenvolveu LPP, observou-se predominância de individuos jovens. Outras publicações nacionais destacam que a população masculina é prevalente em hospitais com referência de trauma, variando de $78 \%$ à $82 \%$; e a incidência de doenças traumáticas, entre as faixas etárias de 15 a 44 anos, chega a 65,1\% 1.9 .

Os pacientes que desenvolveram LPP apresentaram média elevada no escore SAPS III. Os índices de gravidade são importantes indicadores para a identificação do risco de desenvolvimento de LPP e o SAPS III é um instrumento eficaz e satisfatório como prognóstico de gravidade dos pacientes na $\mathrm{UTI}^{10}$.

Em estudos nacionais sobre a incidência de LPP em unidades de terapia intensiva, o tempo de internação foi um fator predisponente para o surgimento de LPP. O aumento no tempo de internação é decorrente do restabelecimento do estado fisiológico e processos orgânicos de pacientes submetidos a procedimentos invasivos para o recondicionamento de órgãos e do controle de doenças associadas ${ }^{1,9}$.

Os pacientes que desenvolveram LPP, neste estudo, apresentaram a integridade cutânea comprometida, em uma média de 2,7 dias de internação, corroborando com outros estudos que descrevem o aparecimento de LPP nos primeiros três dias de internação na UTI ${ }^{1,9}$.

Pacientes críticos com média de balanço hídrico positivo apresentam mais risco para ocorrência de LPP, pela associação do edema com a presença de LPP. O balanço hídrico positivo persistente é relacionado à maior mortalidade na UTI e a repercussões clínicas desfavoráveis como edema de alças intestinais, congestão hepática, edema de miocárdico, congestão pulmonar, edema cerebral, edema renal e edema de tecidos periféricos ${ }^{11}$.

Observou-se no estudo que a aplicação da Escala de Braden é um instrumento importante para o cuidado de enfermagem, pois reforça a importância de avaliação contínua e implementação de medidas preventivas precocemente que favorecem a minimização de problemas futuros das LPP; a aplicação da escala demostrou um preditor de risco para LPP $2,5,12,13$

\section{Limitações do estudo}

Como limitação pode-se apontar o número reduzido de pacientes, o que pode ter interferido na identificação dos possiveis fatores de risco, e o curto período para realização do estudo para desenvolver ações de educação continuada e analisar o seu impacto ao longo do prazo na redução do surgimento de LPP.

\section{Contribuição do estudo para a prática}

Os resultados encontrados favorecem o planejamento de cuidados preventivos específicos para essa clientela que poderão contribuir para melhoria do cuidado e subsídios para o planejamento da assistência de enfermagem e intensificação das estratégias de prevenção. 


\section{CONCLUSÃO}

Constatou-se elevada incidência de LPP, na população em estudo e a multicausalidade dos fatores de risco para o desenvolvimento de LPP nos pacientes críticos com doenças traumáticas. Sugere-se outros estudos para identificar os fatores de risco das LPP e estratégias de fortalecimento de prevenção das lesões, relacionados ao gerenciamento e gestão do cuidado.

\section{Contribuição dos autores:}

Concepção e desenho, análise e interpretação dos dados, redação do artigo, revisãocrítica, revisão final: Carolina Otto, Beatriz Schumacher, Luiz Paulo LemosWiese,Carlos Ferro,Raquel Antonacci Rodrigues.

\section{REFERÊNCIAS}

1. Campanili TCGF, Santos VLCG, Pulido KCS, Thomaz PBM, Nogueira PC. Incidência de úlceras por pressão em pacientes de Unidade de Terapia Intensiva Cardiopneumológica. Rev Esc Enferm USP [Internet]. 2015 [citado 2017 Out 12]; 4으 (Esp): 7-14. Disponivel em: http:// http://www.scielo.br/scielo.php?script=sci_ arttext\&pid=S0080-62342015000700007

2. Loudet CI, Marchena MC, Maradeo MR, Fernández SL, Romero MV, Valenzuela GE, et al. Diminuição das úlceras por pressão em pacientes com ventilação mecânica aguda prolongada: um estudo quasiexperimental. Rev Bras Ter Intensiva. [Internet]. 2017 [citado 2017 Out 12]; 29 (1): 39-46. Disponivel em: http://www.scielo.br/pdf/rbti/ v29nl/0103-507X-rbti-29-01-0039.pdf

3. Irion G. Feridas: novas abordagens, manejo clínico e atlas em cores. $2^{a}$ ed. Rio de Janeiro: Guanabara Koogan; 2012.

4. National Pressure Ulcer Advisory Panel, European Pressure Ulcer Advisory Panel, Pan Pacific Pressure Injury Alliance. Prevention and Treatment of Pressure Ulcers: Quick Reference Guide. 2a ed. Australia: Cambridge Media Osborne Park; 2014.

5. Borghardt AT, Prado TN, Araújo TM, Rogenski NMB, Bringuente MEO. Avaliação das escalas de risco para úlcera por pressão em pacientes criticos: uma coorte prospectiva. Rev. Latino-Amer. Enfermagem [Internet]. 2015 [citado 2015 Out 7]; 23(1): 28-35. Disponivel em: http:// www.scielo.br/pdf/rlae/v23nl/pt_0104-1169-rlae-23-01-00028.pdf

6. Barbosa TP, Beccaria LM, Poletti NAA. Avaliação do risco de úlcera por pressão em UTI e assistência preventiva de enfermagem. Rev Enferm UERJ [Internet]. 2014 [citado 2015 Nov 27]; 22 (3): 353-360. Disponivel em: http://www.facenf.uerj.br/v

22n3/v22n3al0.pdf

7. Goulart LP, Aoki RN, Vegian CFL, Guirardello EB. Carga de trabalho de enfermagem em uma unidade de terapia intensiva de trauma. Rev. Eletr. Enf. [Internet]. 2015 [citado 2017 Out 1]; 16(2): 346-51. Disponivel em: http://dx.doi.org/10.5216/ree.v16i2.22922

8. Brasil. Ministério da Saúde. Secretaria Executiva. Departamento de Informática do SUS. Sistema de Informações sobre Morbidade hospitalar por local de residência. [Internet] 2017. Brasilia: Datasus [2017 Out 17]. Disponivel em: http:// http://tabnet.datasus.gov.br/cgi/ tabcgi.exe?sih/cnv/niuf.def
8. Borghardt AT, Prado TN, Bicudo SDS, Castro DS, Bringuente MEO. Úlcera por pressão em pacientes críticos: incidência e fatores associados. Rev Bras Enferm [Internet]. 2016. [citado 2017 Out 18]; 69(3): 460-7. Disponivel em: http://www.scielo.br/pdf/reben/ v69n3/0034-7167-reben-69-03-0460.pdf

9. Brasil. Ministério da Saúde. Secretaria Executiva. Departamento de Informática do SUS. Sistema de Informações sobre Morbidade hospitalar por local de residência. [Internet] 2017. Brasilia: Datasus [2017 Out 17]. Disponivel em: http:// http://tabnet.datasus.gov.br/cgi/ tabcgi.exe?sih/cnv/niuf.def

10. Bueno HL, Pestana JF. Epidemiologia e validação de escore prognóstico em UTI mista do norte do Paraná. Revista Uningá Review [Internet]. 2015 [citado 2015 Dez 1]; 22(3): 23-29. Disponivel em: http:// www.mastereditora.com.br/periodico/20150601_0

7500l.pdf

11. Avila MON, Rocha PN, Zanetta DMT, Yu L, Burdmann EA. Balanço hidrico, injúria renal aguda e mortalidade de pacientes em unidade de terapia intensiva. J Bras Nefrol [Internet]. 2014 [citado 2015 Dez 6]; 36(3): 379-388. Disponivel em: http://www.scielo.br/pdf/jbn/ v36n3/0101-2800-jbn-36-03-0379.pdf

12. Vasconcelos JMB, Caliri MHL. Ações de enfermagem antes e após um protocolo de prevenção de lesões por pressão em terapia intensiva. Esc Anna Nery [Internet]. 2017 [citado 2017 Out 15]; 21(1) 1-9. Disponivel em: http://www.scielo.br/scielo.php?pid=S1414814520170001002018script=sci_abstractEtlng=pt

13. Castanheiral LS, Alvarenga AW, Correa AR, Campos DMP. Escalas de predição de risco para lesão por pressão em pacientes criticamente enfermos: revisão integrativa.

Enferm. Foco [Internet]. 2018 [citado 2019 Jan 05]; 9 (2): 55-61. Disponivel em: http://revista.cofen.gov.br/index.php/enfermagem/ article/view/1073/446 